\title{
WORD REORDERING ALIGNMENT FOR COMBINATION OF STATISTICAL MACHINE TRANSLATION SYSTEMS
}

\author{
Maoxi Li, Chengqing Zong \\ National Laboratory of Pattern Recognition, Institute of Automation \\ Chinese Academy of Sciences, Beijing
}

\begin{abstract}
Word alignment is a basic and critical process in the Statistical Machine Translation (SMT). The previous work on word alignment mainly focuses on the training process to get the word mapping relation between the source sentences and target sentences. However, the word alignment for combination of SMT system outputs is also important, which aims to find the word correspondence between alternative translation hypotheses of a source language sentence. Unfortunately, it does not attract so much attention in SMT research. In this paper, we propose a novel word alignment approach to effectively address the word alignment between sentences with different valid word orders, which changes the order of the word sequences (called word reordering) of the output hypotheses to make the word order more exactly match the alignment reference. We present experimental results on the IWSLT'2008 challenge tasks with the combination of four state-of-the-art SMT systems outputs. The results show that our approach significantly improves the performance of the system combination.
\end{abstract}

Index Terms - Statistical machine translation, system combination, translation error rate, word error rate, confusion network

\section{INTRODUCTION}

A variety of different paradigms for SMT have been developed, including syntax-based SMT, and phrase-based SMT etc. Each approach has its own strength and weakness. In the recent years, it is attractive to combine the various outputs from multiple SMT systems into a consensus translation. At least, system combination seems to be an expedient powerful means to improve the translation quality in many machine translation tasks empirically.

The overall word-level system combination framework is shown in Figure 1. Alignment approaches in SMT system combination try to find the word correspondences between alternative translation hypotheses of a source language sentence. Unlike word alignment between bilingual sentences, the sentences which need to be aligned in system combination are in the same language, but express the same meaning in different ways or different valid orders, and there may be some translation errors that mask word correspondences. These factors make it hard to identify the alignments. At the same time, a better alignment between translation hypotheses is very important to construct a highquality confusion network while a worse alignment heavily affects the performance of system combination. Therefore the word alignment is a critical process in system combination.

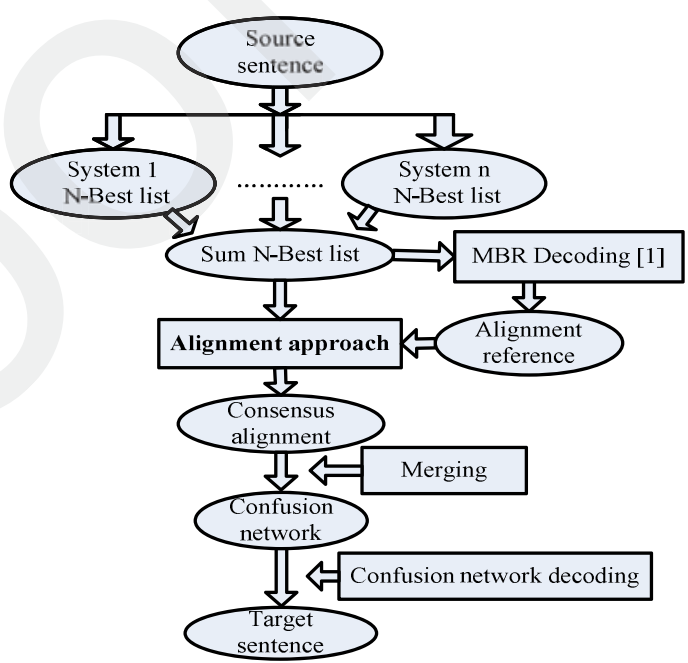

Figure 1: Architecture for word-level system combination

The remainder of the paper is organized as follows: in section 2, we briefly introduce the WER alignment and TER alignment approaches for system combination. In section 3, we describe the word reordering approach in detail. We show our experimental results in section 4 and the final conclusion is drawn in section 5 .

\section{RELATED WORK}

Previous word alignment approaches for word-level system combination include Word Error Rate (WER) alignment [2] approach and Translation Error Rate (TER) [3] alignment [4] approach. The word alignments generated from these approaches are both the byproducts of automatic evaluation measures for machine translation. WER and TER are error metrics for measuring how much two sentences deviate from each other and are based on the edit distance. The edit 
distance between two sentences calculates the minimum numbers of edit operation of words that are necessary to convert one sentence into another.

WER: the edit operations include substitution (Sub), insertion (Ins), and deletion (Del). Specifically:

$$
\operatorname{WER}(E, E r)=\frac{S u b+I n s+D e l}{N} \times 100 \%
$$

where $E$ is the translation hypothesis to be aligned, $E r$ is the alignment reference, $N$ is the total number of words in $E r$.

TER: the allowed edit operations include substitution, insertion, and deletion of a single word as well as shift of word sequences. A shift operation moves a contiguous sequence of words to another location within the hypothesis and has equal cost as the other three edit operations.

Given two translation hypotheses:

$$
\begin{aligned}
& \text { Hyp: this color do you think suits me } \\
& \text { Ref: do you think that color suits me }
\end{aligned}
$$

when the second translation hypothesis is chosen as the alignment reference, the WER alignment and TER alignment may look something like Figure 2 and Figure 3 show, where "null" strings are used to accommodate insertions and deletions.

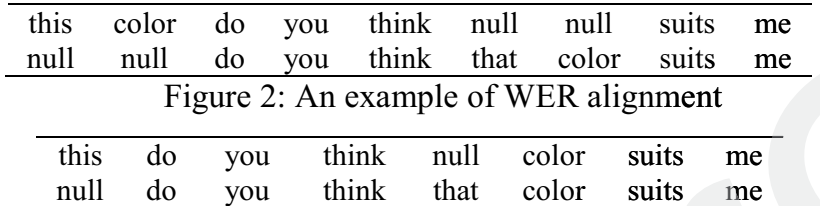

Figure 3: An example of TER alignment

When aligning the sentences with the same word orders the alignment approaches will work effectively for system combination. But if the sentences to be aligned are in different word orders, WER alignment approach completely ignores the differentiation of word order; TER alignment approach tries to solve this problem, but the greedy search algorithm, which is exploited in the open source software tercom script [3], cannot move the word sequences to the proper location in some times because it is too expensive to find an optimal sequence of shifts.

In order to establish a high-quality word alignment between translation hypotheses, we propose a new word alignment approach, namely word reordering alignment (WRA), to set up the words mapping relation between two given translation hypotheses. The alignment approach changes the order of the word sequences of the translation hypotheses to match the order of the alignment reference.

\section{WORD REORDERING ALIGNMENT}

Different from the existing approaches, our WRA approach directly shifts the word sequences of the translation hypothesis to the correct location within the translation hypothesis. In our approach, the continuous word sequences are first found and replaced by some variables. Then we align the variables and words identical to each other in the two sentences and detect the word sequences that should be reordered. Finally, according to some word reordering heuristics, the detected word sequences are shifted to the correct position and dynamic programming are exploited to align the sentences after reordering.

To learn the possible shift units of word sequences, we find the longest common continuous subsequences between the sentences, and substitute some variables for them. For example, when aligning the sentence "this color do you think suits me" to the sentence "do you think that color suits $m e$ ", it looks like "this color $S_{1} S_{2}$ " and " $S_{1}$ that color $S_{2}$ ", where variable $S_{1}$ replaces the word sequences "do you think", variable $S_{2}$ replaces "suits me". In this process, we regard word sequences " $w_{1} x w_{2}$ " and " $w_{1} y w_{2}$ " as the same in order to align the article, such as " $a$ " and "the". where $x$ and $y$ are different single words and replaced by an identical variable. The word subsequences which are replaced by variables are stored in a hash table for restoring the sentences. This is done iteratively until there is no common continuous word subsequence left, which contain no less than two variables and words, among the aligning sentences.

Next, the partial word alignment is created by aligning the identical words and variables in the two sentences, and we find if there are any crosses in the partial alignment words or variables. The cross is a potential possible reordering word sequences.

Mathematically, the cross is defined as:

$$
\left(h_{i}, r_{a_{i}}\right) \in \text { cross if } a_{i}<\max _{j<i} a_{j}
$$

Where $a_{i}$ denotes that the word $h_{i}$ in position $i$ of the translation hypothesis is identical to the word $r_{a_{i}}$ in position $a_{i}$ of the alignment reference. A toy example of word crossing looks like the example in Figure 4, we assume there is a "null" to "null" alignment at left of the first words between sentences to be aligned to aid word reorder.

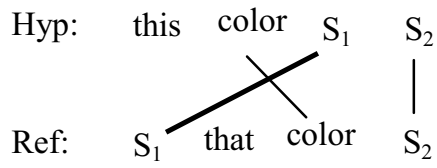

Partial word alignment: 0:0 2:3 3:1 4:4

Figure 4: An example of word crossing

Subsequently, a heuristic approach to reorder the word sequences $h_{i}$ in the cross proceeds as follows: we check whether there exists an adjacent word or variable $r_{a_{j}}$ for word $r_{a_{i}}$ in the alignment reference that has been aligned to a word in the translation hypothesis. If its left (or right) adjacent word or variable $r_{a_{j}}$ maps to the word $h_{j}$ in the translation hypothesis, we shift the word sequences $h_{i}$ to the right (or left) of $h_{j}$ in the translation hypothesis. Note that 
we do not reorder the word sequences in the alignment references; therefore, reordering operations only occur at the translation hypothesis. Meanwhile, if there are not any adjacent words that map to word of translation hypothesis, we have no idea of which position to shift, so we let it alone.

$$
\text { Hyp: S1 this color S2 }
$$

Figure 5: The hypothesis after word reordering

Finally, we substitute the original words for the variables and calculate the most probable alignment between the alignment reference and translation hypothesis after reordering according to the principle of edit distance [5]. Using dynamic programming in a bottom-up fashion, the probable alignment is worked out from the right backwards after calculating the edit distance. The final word reordering alignment between the given hypotheses may look something like:

\begin{tabular}{lllllll}
\hline do & you & think & this & color & suits & me \\
do & you & think & that & color & suits & me \\
\hline
\end{tabular}

Figure 6: An example of word reordering alignment

\section{EXPERIMENT RESULTS}

\subsection{Experimental setting}

We carried out the experiments on IWSLT'2008 challenge tasks, which include Chinese-to-English (CE) and Englishto-Chinese (EC) automatic speech recognition outputs (ASR) translation tasks as well as correct recognition result transcripts (CRR) translation tasks. For ASR translation tasks, every spontaneous speech sentence was recognized by multiple ASR engines and N-Best $(N=5 \sim 20)$ list hypotheses for each speech sentence were provided, while for CRR translation tasks, a correct recognition result transcripts was provided for each speech sentence. Table 1 shows the corpus statistics of the tasks, including a training corpus and four test corpora. The training corpus contains sentence pairs of basic traveling expression with case and punctuation information.

In our experiments, we take the translation outputs from four SMT systems including phrase-based SMT systems CASIA [6], Moses [7], maximum entropy based phrase reordering SMT system similar to [8] (MEBTG), and a hierarchical phrase-based SMT system similar to [9] (Hiero). Each system generates 10-best list for each CRR source sentences and 1-best for each ASR output hypotheses. System combination results are presented based on three different word alignment strategies: WER alignment (WER), TER alignment (TER), and our approach WRA.

For CE translation tasks, the first word in each N-Best list hypotheses is written in upper case, a reordering of words between translation hypothesis and alignment reference may lead to conflicting cases, therefore, everything is lowercased. Unless otherwise stated, performance was measured using BLEU [10] score based on case insensitivity.

Confuse network decoding features include normalized word voting score, language model score, word penalty. The decoding process may be written as

$$
\mathrm{e}^{*}=\underset{\mathrm{e}}{\operatorname{argmax}}\left(\lambda_{\mathrm{AL}} \log \mathrm{P}_{\mathrm{AL}}+\lambda_{\mathrm{LM}} \log \mathrm{P}_{\mathrm{LM}}+\lambda_{\mathrm{WP}} \log \mathrm{P}_{\mathrm{WP}}\right)
$$

where the probability $\mathrm{P}_{\mathrm{AL}}, \mathrm{P}_{\mathrm{LM}}, \mathrm{P}_{\mathrm{wP}}$ represent normalized word voting score, language model score, and word penalty. The parameters $\lambda_{\mathrm{AL}}, \lambda_{\mathrm{LM}}, \lambda_{\mathrm{WP}}$ represent their weights respectively and constrained to sum to 1 . The argmax operation denotes the search problem, that is, the generation of the consensus translation after combining the N-Best list hypotheses from multiple SMT systems and $\mathrm{e}^{*}$ is the final output target sentence. In our experiments, the weights are manually set as followed:

$$
\lambda_{\mathrm{AL}}=0.55, \lambda_{\mathrm{LM}}=0.10, \lambda_{\mathrm{WP}}=0.35
$$

\begin{tabular}{|c|c|c|c|}
\hline \multirow{4}{*}{ data } & Chinese & English \\
\hline \multirow{4}{*}{ CT_EC ASR } & Sentences & \multicolumn{2}{|c|}{$20 \mathrm{k}$} \\
\cline { 2 - 4 } & Words & $172 \mathrm{k}$ & $153 \mathrm{k}$ \\
\cline { 2 - 4 } & Tokens & $235 \mathrm{k}$ & $653 \mathrm{k}$ \\
\cline { 2 - 4 } & Sentences & \multicolumn{2}{|c|}{251} \\
\cline { 2 - 4 } & Words & $1.4 \mathrm{k} \times 7$ & $2.6 \mathrm{k}$ \\
\hline \multirow{4}{*}{ CT_CE ASR } & Tokens & $3.4 \mathrm{k} \times 7$ & $8.9 \mathrm{k}$ \\
\cline { 2 - 4 } & Wontences & \multicolumn{2}{|c|}{500} \\
\cline { 2 - 4 } & Tokens & $18.7 \mathrm{k}$ & $20.3 \mathrm{k} \times 7$ \\
\hline \multirow{3}{*}{$\begin{array}{c}\text { CT_EC } \\
\text { CRR }\end{array}$} & Sentences & \multicolumn{2}{|c|}{251} \\
\cline { 2 - 4 } & Words & $1.4 \mathrm{k} \times 7$ & $1.6 \mathrm{k}$ \\
\cline { 2 - 4 } & Tokens & $3.4 \mathrm{k} \times 7$ & $5.4 \mathrm{k}$ \\
\hline \multirow{3}{*}{ CE CRR } & Sentences & \multicolumn{2}{|c|}{489} \\
\cline { 2 - 4 } & Words & $3.4 \mathrm{k}$ & $3.2 \mathrm{k} \times 6$ \\
\cline { 2 - 4 } & Tokens & $10.8 \mathrm{k}$ & $8.8 \mathrm{k} \times 6$ \\
\hline
\end{tabular}

Table 1: Corpus statistics for English-Chinese and Chinese-English translation tasks

\subsection{Results}

For ASR translation tasks, we translate the N-Best list hypotheses of a spontaneous speech sentence and produced 1 -Best for each. Actually, $4^{*} N$ best hypotheses for each speech sentence take part in system combination, here 4 denotes the four translation systems. The CT_EC test set is IWSLT'2008 CT_EC N-Best Dev Set, while the CT_CE test set is IWSLT'2008 CT_CE N-Best Dev Set 5. Table $\overline{2}$ shows the system combination results. We find that system combination based on WER alignment improves over the best baseline system by 0.38 BLEU point on CT CE task and 0.07 BLEU point on CT_EC task. System combination 
based on TER alignment further improves the BLEU score by 0.79 on CT_CE task and 0.74 on CT_EC task. We observe additional 0.02 BLEU point on $\mathrm{CT}_{-} \mathrm{CE}$ task and 0.44 BLEU point on CT_EC task are achieved with system combination based on WRA. To summarize, exploit WRA for system combination, we achieve 1.19 BLEU point improvement on CT_CE task and 1.25 BLEU point improvement on CT_EC task over the best baseline system.

\begin{tabular}{|c|c|c|}
\hline \multirow{2}{*}{ System } & CT_CE & CT_EC \\
\cline { 2 - 3 } & BLEU[\%] & BLEU[\%] \\
\hline Hiero & 17.57 & 29.37 \\
CASIA & 20.45 & 28.45 \\
MEBTG & 20.73 & 29.45 \\
Moses & $\mathbf{2 2 . 1 2}$ & $\mathbf{3 0 . 3 1}$ \\
\hline WER & 22.50 & 30.38 \\
TER & 23.29 & 31.12 \\
WRA & $\mathbf{2 3 . 3 1}$ & $\mathbf{3 1 . 5 6}$ \\
\hline
\end{tabular}

Table 2: System combination results with different alignment approaches on ASR translation tasks

Table 3 shows the system combination results on CE and CT_EC CRR translation tasks. The CE test set is IWSLT'2007 CE Test Set, while the EC test set is IWSLT'2008 CT_EC 1-Best Dev Set. We consistently observe that system combination based on WRA improves over the best baseline by 0.88 BLEU point on CE task and 0.58 BLEU point on CT EC task.

\begin{tabular}{|c|c|c|}
\hline \multirow{2}{*}{ System } & CT_CE & CT EC \\
\cline { 2 - 3 } & BLEU[\%] & BLEU[\%] \\
\hline CASIA & 25.99 & 35.20 \\
Moses & 28.01 & 34.37 \\
MEBTG & 30.20 & $\mathbf{3 5 . 3 6}$ \\
Hiero & $\mathbf{3 1 . 5 2}$ & 33.96 \\
\hline WER & 31.38 & 35.40 \\
TER & 32.32 & 35.59 \\
WRA & $\mathbf{3 2 . 4 0}$ & $\mathbf{3 5 . 9 4}$ \\
\hline
\end{tabular}

Table 3: System combination results with different alignment approaches on CRR translation tasks

\section{CONCLUSIONS AND FUTURE WORK}

Our WRA approach effectively addresses the word alignment between sentences with different valid word order. Furthermore, system combination based on WRA approach significantly improves the translation quality than the approaches of system combination based on WER alignment or TER alignment.

However, in this paper, the weights of confuse network decoding features are set manually. Our future work will focus on tuning the weights with some discriminative optimization technique.
Another potential area for improvement is to set up the word alignment with the help of information from synonymous words and part-of-speech of the hypotheses words.

\section{ACKNOWLEDGEMENTS}

The research work has been funded by the Natural Science Foundation of China under Grant No.60575043 and 60736014, the National Key Technology R\&D Program under Grant No. 2006BAH03B02, the Hi-Tech Research and Development Program ("863" Program) of China under Grant No. 2006AA01Z194, 2006AA010108-4, and Nokia Research Center, Beijing as well.

\section{REFERENCES}

[1] S. Kumar and W. Byrne, "Minimum Bayes-risk decoding for statistical machine translation," in Proc. of HLT, 2004.

[2] L. Mangu, E. Brill, and A. Stolcke, "Finding consensus among words: Lattice-based word error minimization," in Proc. Eur. Conf. Speech Commun. Technol, 1999.

[3] M. Snover, B. Dorr, R. Schwartz, L. Micciulla, \& J. Makhoul, "A study of translation edit rate with targeted human annotation," in Proc. Assoc. for Machine Trans. in the American, 2006.

[4] K.C. Sim, W.J. Byrne, M.J.F. Gales, H. Sahbi \& P.C. Woodland, "Consensus network decoding for statistical machine translation system combination," in proc. ICASSP, volume 4, pages 105-108.

[5] O. Kemal, "Error-tolerant finite-state recognition with application to morphological analysis and spelling correction," Computational Linguistics, 22(1):73-89.

[6] Y. Zhou, Y. He, and C. Zong, "The CASIA phrase-based statistical machine translation system for IWSLT 2007," in proc. International Workshop on Spoken Language Translation, 2007.

[7] P. Koehn, H. Hoang, A. Birch, C. Callison-Burch, M. Federico, N. Bertoldi, B. Cowan, W. Shen, C. Moran, R.Zens, C. Dyer, O. Bojar, A. Constantin and E. Herbst, "Moses: open source toolkit for statistical machine translation", in proc. ACL, 2007.

[8] D. Xiong, Q. Liu, and S. Lin, "Maximum entropy based phrase reordering model for statistical machine translation," in proc. of COLING-ACL, 2006.

[9] D. Chiang, "A hierarchical phrase-based model for statistical machine translation," in Proc. ACL, 2005.

[10] K. Papineni, S. Roukos, T. Ward, and W. Zhu, "BLEU: a method for automatic evaluation of machine translation," Tech.Rep. RC22176 (W0109-022), IBM Research Division, 2001. 